\title{
Flexible Error Concealment for H.264 Based on Directional Interpolation
}

\author{
Olivia Nemethova, Ameen Al-Moghrabi and Markus Rupp \\ TU Wien, Institute of Communications and RF Engineering \\ Gusshausstrasse 25/389, 1040 Wien Austria \\ \{onemeth, mrupp\}@nt.tuwien.ac.at
}

\begin{abstract}
The losses of packets cannot be avoided if real-time video is transported over error prone environments. To conceal missing parts of video pictures, the spatial and temporal correlation feature of natural video sequences is used. However, in some cases - for instance in case of a scene change - there is no temporal correlation available and thus spatial error concealment has to be used. This article proposes a flexible spatial error concealment based on directional interpolation method that performs well also if only two neighboring boundaries are used as common for H.264 spatially predicted frames. The proposed method was implemented and tested in a H.264 codec together with other error concealment methods to evaluate their performance.
\end{abstract}

\section{Introduction}

H.264 [1] is the newest high compression digital video codec standard written by the ITU-T Video Coding Experts Group (VCEG) together with the ISO/IEC Moving Picture Experts Group (MPEG). Thanks to its new features, H.264 achieves higher compression gain than H.263 and MPEG-4 while preserving the same visual quality. This makes H.264 well suited for transmission over bandwidth limited systems, like mobile wireless systems. To match the screen of a mobile terminal, a $144 \times 176$ pixel resolution (also called QCIF) is used. For PDAs and Laptops usually CIF $(288 \times 352$ pixel $)$ is used. In such small resolutions every single pixel can carry a lot of information.

Real-time video is usually transmitted via unreliable User Datagram Protocol (UDP) without any possibility for retransmissions at the transport layer. Each UDP packet contains Cyclic Redundancy Check (CRC) allowing error detection. If a CRC fails, the whole UDP packet is discarded. A UDP packet represents usually a rather large part of the picture and its loss results in considerable visual perceptual quality distortion. To conceal the distortion, interpolation methods in temporal, spatial or spatial-frequency domain are used [2].
The optimal method depends on many factors: character of the lost region (smooth, edges, texture), amount of instantaneous motion in the video sequence, presence of scene change, region of interest, etc. In case of small resolution videos with low amount of movement and without scene changes, temporal interpolation would surely be the best candidate for error concealment. The reason is that the missing parts of the picture contain information that can be spatially almost uncorrelated with the correctly received parts of the picture. Such missing parts (for instance the whole eye of a talking head) cannot be recovered by spatial interpolation only. However, there are cases, where it is not efficient to use temporal interpolation. Temporal error concealment is not efficient if non-linear movement is present in the sequence, and if there is fast motion or sudden color change. Serious visual artifacts may occur if temporal concealment is applied after the scene changes. Due to the temporal prediction used in the compression process, the error propagates from frame to frame until a temporal independent frame (I frame) arrives successfully. Scene changes appear often for instance in sport games, video clips, cinema trailers, news which represent popular content for mobile devices. Therefore, in [3] an error concealment method is chosen according to the decision of a scene change detector. However, in [3] the very simple, but not very well performing spatial concealment method is used. Thus, the performance of postprocessing reduces considerably as soon as spatial concealment has to be used. To fully exploit the spatial correlation of natural pictures, methods preserving the edges should be used.

The intention of this article is to propose and evaluate a flexible and simple spatial domain interpolation method that preserves edges and smoothes along them. In Section 2 previous work is reviewed and the problem is formulated. Section 3 presents edge detection mechanism used, while in Section 4 proposed methods are introduced. In Section 5 the results are presented and interpreted. Section 6 contains conclusions and some final remarks. 


\section{Problem Formulation}

\subsection{Smooth recovery}

The simplest and commonly used spatial domain interpolation method is weighted averaging [2]. According to this method each pixel of a missing macro block is interpolated as a linear combination of the nearest pixels in the macro block boundaries. Each pixel $p(i, j)$ is calculated as follows:

$$
p(i, j)=\frac{d_{R} p_{L}+d_{L} p_{R}+d_{B} p_{T}+d_{T} p_{B}}{d_{L}+d_{R}+d_{T}+d_{B}},
$$

where $d_{L}, d_{R}, d_{T}, d_{B}$ are the distances between the interpolated pixel and the $p_{L}=p(0, j), p_{R}=p(N+1, j)$, $p_{T}=p(i, N+1), p_{B}=p(i, 0)$ are the nearest pixels in all four boundaries as shown in Figure 2; $N$ is the size of macro block. Please note, that $p(1,1)$ we put in the left bottom corner of a $N \times N$ macro block, first coordinate being in horizontal and second in vertical direction. Weighted averaging only performs well if the missing block was smooth, otherwise it produces visible artifacts as shown in Figure 1.
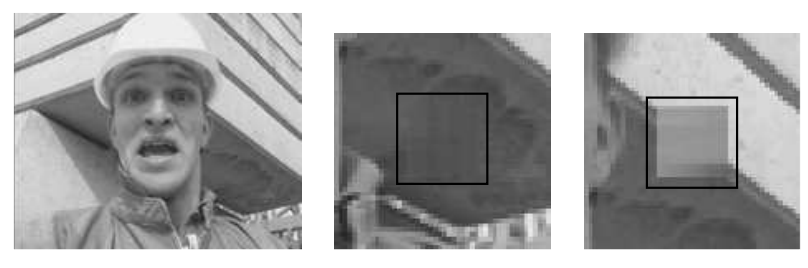

Figure 1: Interpolation by weighted averaging (from left to right): original picture, weighted averaging recovery applied to smooth region, weighted averaging applied to region with an edge.

\subsection{Preserving edges}

Other methods were investigated, that prolong edges entering the missing blocks and smooth along them. In [4] an iterative method based on projection onto convex sets (POCS) is presented. Edges are reconstructed iteratively in spatial-frequency domain by means of a band-pass filter adapted to the neighborhood of the damaged blocks. However, this method is rather complex and therefore not really suitable for real-time wireless transports. An alternative is interpolation in spatial or spatial-frequency domain as proposed in [5]. The edge is first classified in one of four main directions and then smoothing in the identified direction is performed. The drawback of this method is, that it supports only one main direction for the whole missing block. While this is possibly sufficient for higher resolution, it brings limited improvement for low resolutions where often more edges meet in one missing area. In [6] a method considering all detected edges is proposed. This method matches the edges entering and leaving the missing area, partitions the area accordingly and smoothes preserving the edges. Unfortunatelly, the matching of edges can not be always performed reliably. Furthermore, such method requires all four boundaries.

\subsection{H.264 requirements}

All four boundaries can be used if there is only a single macro block or block area missing. This is seldom the case for video transmissions over packet networks. The size and shape of the missing area is determined by the chosen slicing mode [1]. Appropriate detection mechanism can also reduce the size of the missing area [7]. The situation where all four boundaries can be used occurs in H.264 only for temporal predicted frames. For spatially predicted frames, the right and bottom macro blocks may use the missing block for prediction and therefore cannot be used for the error concealment. Usage of the spatial prediction represents the main challenge for the error concealment due to the possible spatial error propagation. If error concealment is not performed sufficiently well, large visual impairments may occur in the reconstructed picture.

Thus, the requirements for an efficient spatial error concealment method are the following:

- ability to distinguish and recover more than one edges crossing the missing block,

- flexibility with respect to the number of boundaries that can be used,

- acceptable performance for different sizes and shapes of missing area,

- low computational complexity supporting real time applications,

- high quality of reconstruction especially for the I frames.

\section{Edge Detection}

An efficient and widely used method for edge detection is calculating the gradient field by means of the vertical and horizontal Sobel mask:

$$
\mathbf{S}_{x}=\left[\begin{array}{ccc}
-1 & 0 & 1 \\
-2 & 0 & 2 \\
-1 & 0 & 1
\end{array}\right], \quad \mathbf{S}_{y}=\left[\begin{array}{ccc}
1 & 2 & 1 \\
0 & 0 & 0 \\
-1 & -2 & -1
\end{array}\right]
$$

The masks are applied on luminance value $y_{i, j}$ of each pixel at the missing area boundaries as follows: 


$$
\begin{aligned}
G_{x}(i, j) & =\operatorname{vec}\left(\mathbf{S}_{x}\right)^{T} \operatorname{vec}(\mathbf{F}(i, j)), \\
G_{y}(i, j) & =\operatorname{vec}\left(\mathbf{S}_{y}\right)^{T} \operatorname{vec}(\mathbf{F}(i, j)),
\end{aligned}
$$

$\mathbf{F}(i, j)$ being the relevant part of a boundary:

$$
\mathbf{F}(i, j)=\left[\begin{array}{ccc}
y_{i-1, j-1} & y_{i-1, j} & y_{i-1, j+1} \\
y_{i, j-1} & y_{i, j} & y_{i, j+1} \\
y_{i+1, j-1} & y_{i+1, j} & y_{i+1, j+1}
\end{array}\right] .
$$

Please, note that to reduce complexity it is possible to use luminance values of pixels only as chrominance is usually smoother. However, [8] recommends to use both - luminance and chrominance to improve the performance. Magnitude of the gradient $|G(i, j)|$ and its direction $\theta_{g}(i, j)$ can then be calculated for each pixel at the missing block boundary as follows:

$$
\begin{array}{r}
|G(i, j)|=\sqrt{G_{x}^{2}(i, j)+G_{y}^{2}(i, j)}, \\
\theta_{g}(i, j)=\arctan \left(\frac{G_{y}(i, j)}{G_{x}(i, j)}\right) .
\end{array}
$$

Please, note that the slope $a(i, j)$ of the edge, perpendicular to the gradient direction $\theta_{g}$, can be expressed as follows:

$$
a(i, j)=\frac{G_{y}(i, j)}{G_{x}(i, j)}=\cot \theta_{g}(i, j) .
$$

The dominant gradient direction within certain area $A$ can be determined as the sum of all pixel gradients in $A$ weighted by their magnitude:

$$
\theta_{g d}=\frac{\sum_{\forall i, j \in A} \theta_{g}(i, j)|G(i, j)|}{\sum_{\forall i, j \in A}|G(i, j)|} .
$$

A threshold can be set to consider only the edges with magnitude above certain values. In our implementation we used an empirically set fixed threshold. An adaptive threshold set according to the content could even more improve the results. The gradient is usually calculated for each pixel of several pixel wide boundary in all blocks neighboring to the missing one. Computational complexity (but the quality of detection as well) can be decreased by taking narrower boundaries or taking a subset of pixels only to calculate the gradient.

\section{Interpolation and Partitioning}

\subsection{Main direction smoothing}

After detecting the edges at the missing area boundary, these should be further prolonged. The simplest approach is to support one main edge direction $\theta_{e d}$ (with corresponding edge slope $a_{d}=\cot \theta_{e d}$ ) per lost block only and interpolate in that direction by means of weighted averaging:

$$
p(i, j)=\frac{1}{d_{1}+d_{2}}\left[d_{2} p_{1}+d_{1} p_{2}\right],
$$

where $p_{1}$ and $p_{2}$ are the points in boundaries from which the missing pixel is interpolated. They can be obtained as an intersection of block boundaries with line having slope $a_{d}$, including $p(i, j)$. Symbols $d_{1}$ and $d_{2}$ denote the distance of $p(i, j)$ from $p_{1}$ and $p_{2}$ respectively:

$$
\begin{array}{r}
p_{1}=p\left(i_{1}, j_{1}\right), \quad p_{2}=p\left(i_{2}, j_{2}\right), \\
i_{1}=\max \left[i-i \cdot \frac{1}{a_{d}} ; 0\right], \\
j_{1}=\max \left[j-j \cdot a_{d} ; 0\right], \\
i_{2}=\min \left[i+i \cdot \frac{1}{a_{d}} ; N+1\right], \\
j_{2}=\min \left[j+j \cdot a_{d} ; N+1\right], \\
d_{1}=\sqrt{\left(i-i_{1}\right)^{2}+\left(j-j_{1}\right)^{2}}, \\
d_{2}=\sqrt{\left(i+i_{2}\right)^{2}+\left(j+j_{2}\right)^{2}} .
\end{array}
$$

Please, note, that the coordinates have to be truncated to integer values. This method together with the weighted averaging over smooth regions is visualized in Figure 2.
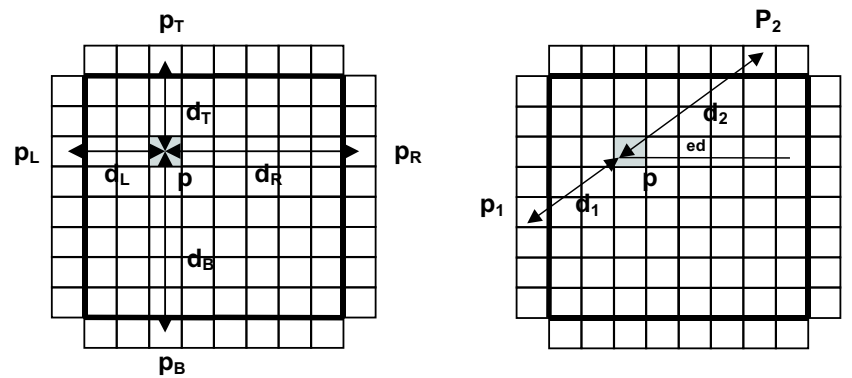

Figure 2: Spatial interpolation: weighted averaging (left), main direction smoothing (right).

The quality of interpolation depends strongly on the resolution of the edge direction. This method does not provide satisfying results if there are more dominant edges entering the missing area, as shown in Figure 3.

In natural scene pictures with resolution as small as QCIF, there are still many blocks with more than one dominant edge. In such case it is important to decide how the edges will be prolonged. Prolonging the edges results in partitioning of the missing block, each partition can be further recovered by smoothing. 

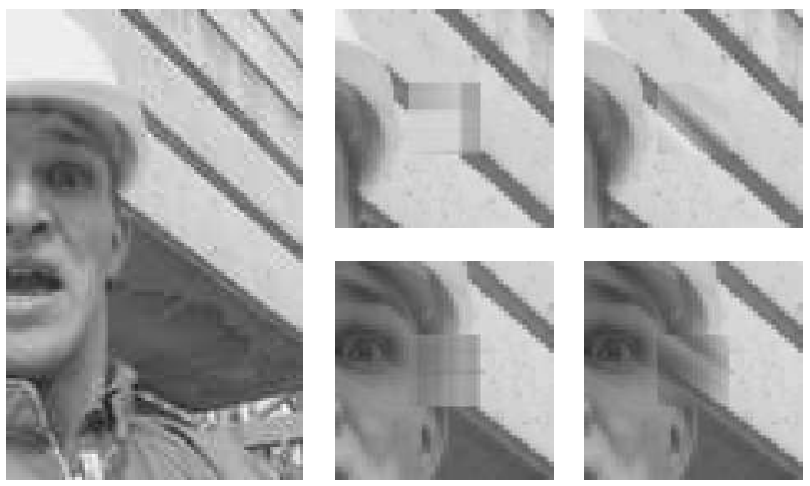

Figure 3: Relevant part of original picture (left), weighted averaging recovery (middle), main direction smoothing recovery(right)

\subsection{Partitions smoothing}

There are two possibilities: either an edge enters and leaves the missing block, or it ends inside. In [6] an algorithm is proposed that partitions the missing block area accordingly. However, it is not trivial to distinguish between the pairs of edges that enter and leave the blocks. Moreover, if only left and top boundaries are known, there is no possibility to know whether an edge leaves the block or not (unless it crosses the left and top boundary only). This ambiguity is shown in Figure 4 .
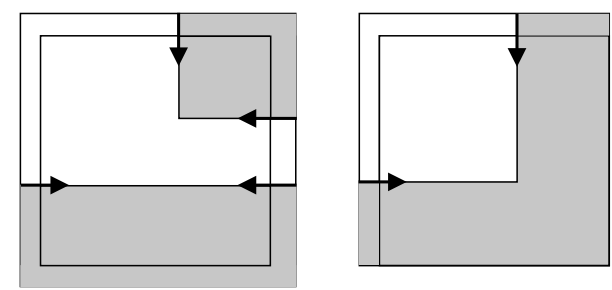

Figure 4: Distinguishing intersections if all four neighbors (left) and only two of them (right) are known.

There is no possibility to avoid the ambiguity if we cannot use any information from bottom and right neighbors or from previous/consecutive frames. Therefore, we investigate other approaches for partitioning and interpolation. The intention is to subdivide missing blocks into regions of dominance - each such region supporting one main edge direction. Interpolation is then similar as in the previous section, applied to each particular region. In Figure 5 possible subdivision into four and eight regions of dominance is shown together with corresponding results of artificial picture recovery. Natural scene recovery can be seen in Figure 6 .

Fixed partitioning with more partitions than necessary may cause some discontinuities. This effect can be easily avoided by variable partitioning as shown in Figure 7 .
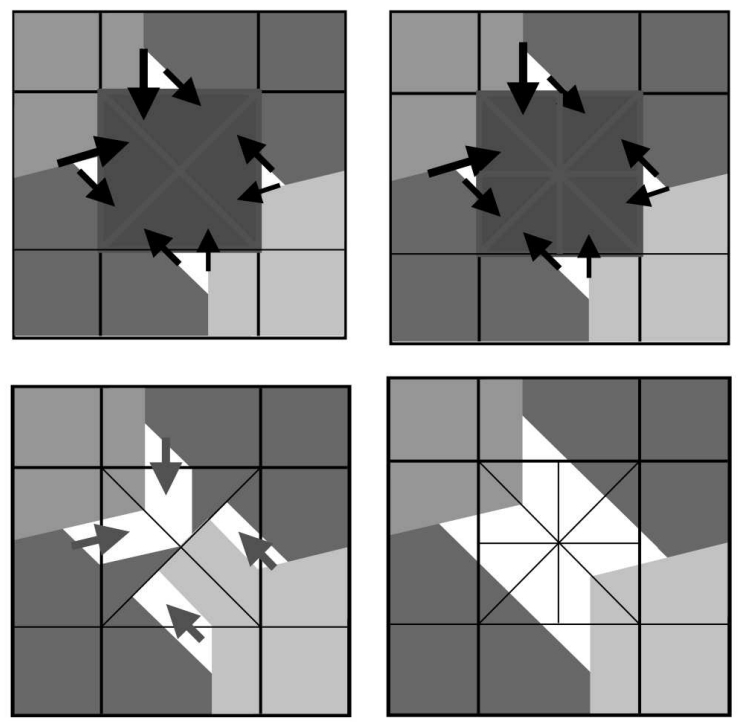

Figure 5: On the top: four (left) and eight (right) fixed regions of dominance; on the bottom: recovery of artificial picture using four (left) and eight (right) fixed regions of dominance.
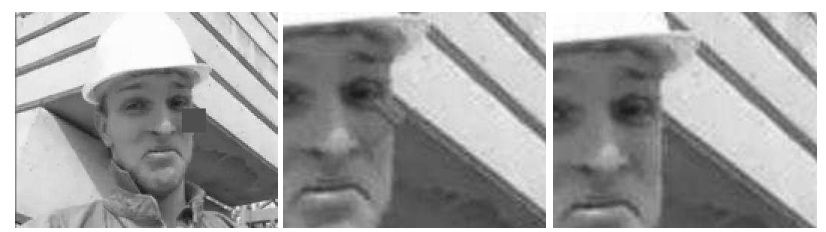

Figure 6: From left to right: Original picture with missing block, recovery with four fixed size partitions, recovery with eight fixed size partitions.

To obtain efficient variable size partitioning, following steps have to be performed:

- Segment missing blocks into M partitions of four, eight or more according to the missing block size. Eight basis regions for $16 \times 16$ blocks represent a good tradeoff between the complexity and efficiency.

- Calculate the main direction for each of the partitions.

- Decide according to a predefined threshold, which partitions possess clear edges.

- Attach partitions without clear edges to those with clear edges that correlates most to their detected direction. 

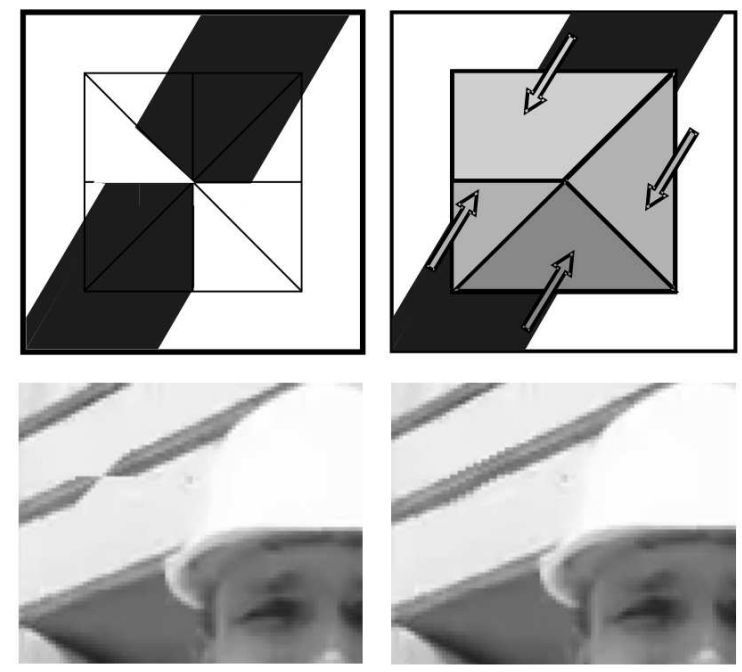

Figure 7: Discontinuities caused by fixed partitioning (left) and variable size partitioning (right).

\section{Results}

\subsection{Experimental setup}

We implemented the error concealment methods into the Joint Model (JM) H.264 software [9]. We added spatial error concealment using weighted averaging and both proposed fixed and flexible partitioning directional interpolation methods. All methods are executed at runtime. Since the error concealment is performed during the decoding, only two neighboring blocks can be used for recovery. Concealed blocks in an I frame are further used for spatial (intra) prediction of the following blocks; the whole I or P frame containing concealed blocks is further used for temporal prediction. In our implementation, the concealed blocks can be further used for error concealment (if they are neighbors of a missing block). To evaluate the performance we encoded the foreman sequence with slicing mode 0 (frame $=$ slice). The I frame frequency was 20 , both $\mathrm{P}$ and $\mathrm{B}$ frames were used. To evaluate the proposed method, we used spatial error concealment for all types of frames. The only exception is the first block, which does not have any usable neighbors and therefore needs to be concealed by a temporal domain error concealment. In praxis, spatial error concealment would only be needed in case of a scene change or very high motion (especially for temporal predicted blocks).

\subsection{Evaluation}

To compare the global performance of implemented methods, we first introduced uniformly distributed macro block losses $\left(P_{\text {loss }}=0.05\right)$ into the stream and then concealed the missing blocks using the directional interpolation from the top and left neighbor, and the weighted averaging. The results by means of luminance Peak to Signal-to-Noise Ratio (PSNR) over frame number for the first 40 frames of the foreman sequence can be seen in Figure 8.

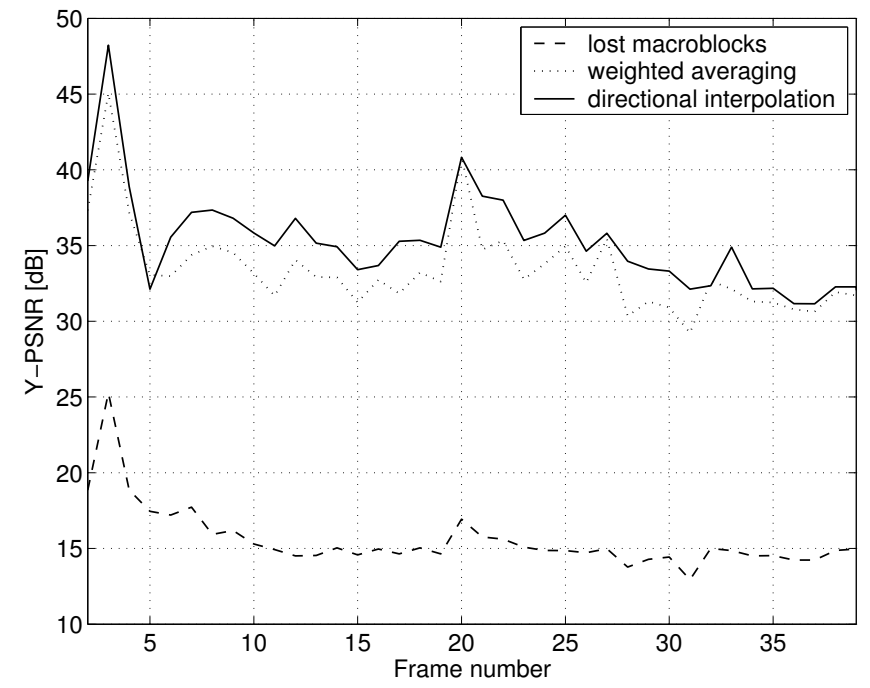

Figure 8: PSNR over frame number for weighted averaging and proposed directional interpolation.

In average, proposed spatial error concealment method is approximately $1-2 \mathrm{~dB}$ better than the weighted averaging, depending on the position of the error. The reconstruction is especially difficult for the I frames because any difference between the lost original block and its concealed version results in non exact spatial and temporal prediction, which may lead to considerable visual artifacts. In our experiments, the proposed method clearly outperforms weighted averaging for the temporal predicted frames. For spatially predicted frames, directional interpolation also performs better than the weighted averaging; however, the gain gets smaller for complicated scenes containing a lot of edges. The most limiting factor is the ambiguity of the edges described in Section 4.2. Please note, that the PSNR does not always correspond to the visual perceptual quality as seen by user. Some screenshots of the concealed foreman sequence when using two neighbors only for an I and a $\mathrm{P}$ frame are presented in Figure 9.

On the left side in the figure, there is a small mismatch of the edge direction caused by the interpolation that uses two neighbors only. On the right side, the I frame was concealed. In the original picture in the top, error propagation caused by spatial prediction used at the encoder can be seen and its reduction when using error concealment. However, the error propagation could not be completely avoided because the concealed block is not identical with the original one, from which the in- 

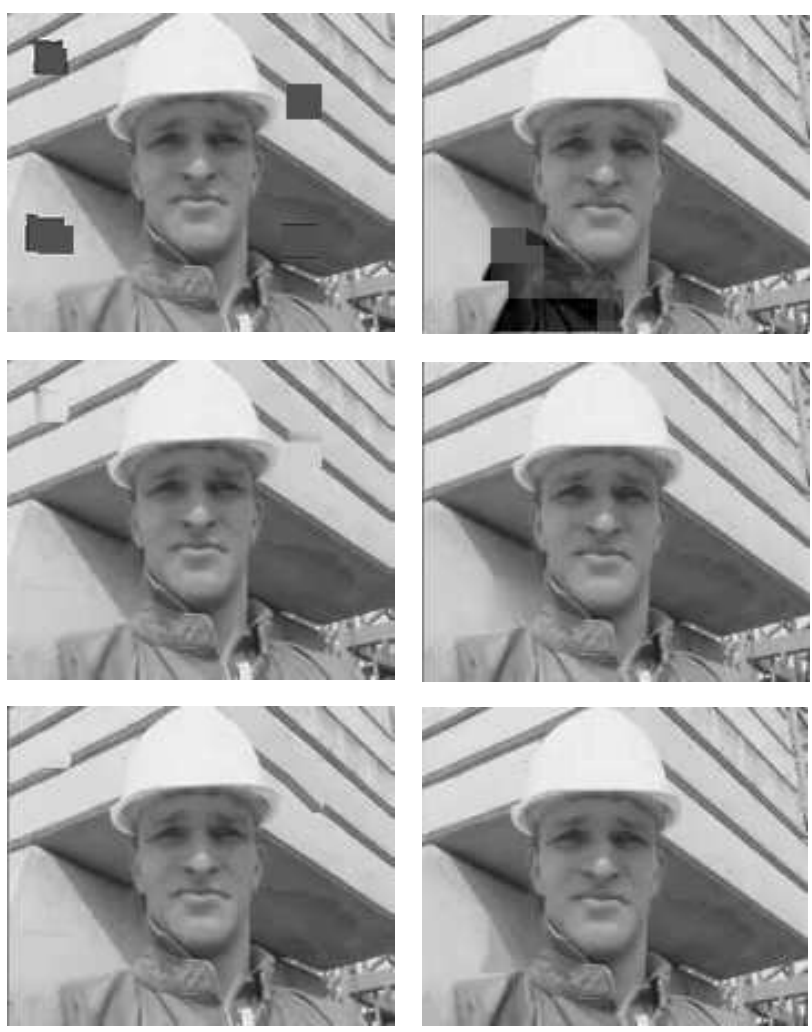

Figure 9: Screenshots of the foreman sequence (from top to bottom): original with missing blocks, concealed with weighted averaging, concealed with proposed method.

tra prediction was derived. The resolution of the edge detector also plays a very important role as can be seen in Figure 10. In general, spatial error concealment for low resolutions is rather challenging task. The proposed flexible partitioning method performs well in most of the cases, but still, there is a limitation of weaker spatial correlation, especially for video sequences with high amount of spatial information. For two neighboring blocks only, the task of spatial interpolation becomes ambiguous - we can not know whether an edge goes through the block or finishes there. Therefore also small discontinuities may occur especially if the edges crossing the block are not really linear.

\section{Conclusions}

Our intention was to propose a set of low complexity error concealment methods for H.264 that would improve the user visual perceptual quality after the losses in a mobile network. For QCIF resolution the best way to recover the information is temporal concealment. However, there are situations where no temporal concealment can be used as for example after a scene change. For such cases spatial error concealment has to be applied for both, spatially and temporal predicted frames. In
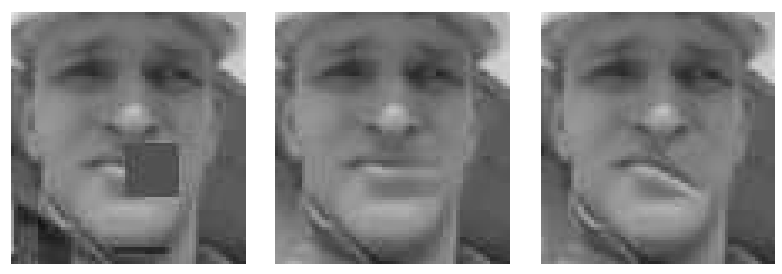

Figure 10: Prolonging edges: original with missing block, concealed with weighted averaging, concealed with directional interpolation using two neighbors only and small edge direction resolution.

this article we discussed the design of the spatial error concealment for H.264, based on directional interpolation and preserving the edges. We proposed and implemented into the H.264 decoder simple and scalable algorithms one with fixed, another one with flexible partitioning that can be used either if two or four blocks neighboring to the missing one are known. The proposed algorithm performs well compared to alternative spatial concealment strategies while keeping the computational costs reasonable so that it can be used also in real-time video streaming application.

\section{References}

[1] T. Wiegand, G.J. Sullivan, G. Bjontegaard, A. Luthra, "Overview of the H.264/AVC Video Coding Standard," IEEE Trans. on Circuits and Systems for Video Technology, vol. 13, no. 7, pp. 560-576, July 2003.

[2] M.T. Sun, A.R. Reibman, "Compressed Video over Networks," Signal Processing and Communications Series, Marcel Dekker Inc., New York, 2001.

[3] G. Gennari, G.A. Mian, L. Celetto, "A H.264 Decoder Robust to Transmission Errors," Proc. of EUSIPCO, vol. 1, pp. 114-120, Vienna, Austria, Sep. 2004.

[4] H. Sun, W. Kwok, "Concealment of Damaged Block Transform Coded Images Using Projections onto Convex Sets," IEEE Transactions on image Processing, vol. 4, no. 4, Apr. 1995.

[5] J.W. Suh, Y.S. Ho, "Error Concealment based on Directional Interpolation," IEEE Trans. on Consumer Electronics, vol. 43, no. 3, pp. 295-302, Aug. 1997.

[6] W.Y. Kung, C.S. Kim, C.J. Kuo, "A Spatial-Domain Error Concealment Method With Edge Recovery And Selective Directional Interpolation," Proc. of ICASSP, 2003.

[7] C. Weidmann, P. Kadlec, O. Nemethova, A. Al-Moghrabi, "Combined Sequential Decoding and Error Concealment of H.264 Video," Proc. of MMSP, Oct. 2004, Siena, Italy.

[8] A. Koschan, M. Abidi, "Detection and Classification of Edges in Color Images," IEEE Signal Processing Magazine, vol. 22, no. 1, Jan. 2005.

[9] H.264/AVC Software Coordination, "JM Software," ver.7.3, available in http://iphome.hhi.de/suehring/tml/. 